\title{
On the Core of Games with Communication Structures
}

\author{
by \\ M. Josune Albizuri \\ and \\ Peter Sudhölter
}

Discussion Papers on Business and Economics

No. $6 / 2014$

FURTHER INFORMATION

Department of Business and Economics Faculty of Business and Social Sciences University of Southern Denmark Campusvej 55 DK-5230 Odense M Denmark

Tel.: +4565503271

Fax: +45 65503237

E-mail: lho@sam.sdu.dk

http://www.sdu.dk/ivoe 


\title{
On the Core of Games with Communication Structures*
}

\author{
M. Josune Albizuri ${ }^{\dagger} \quad$ Peter Sudhölter ${ }^{\ddagger}$
}

March 9, 2014

\begin{abstract}
It is well-known that the core on several domains of cooperative transferable utility (TU) and nontransferable utility (NTU) games is characterized by various combinations of axioms containing some versions of the reduced game property, of its converse, or of the reconfirmation property with respect to the Davis-Maschler reduced game. We show that these characterizations are still valid for games with communication structures à la Myerson when using the notion of the reduced communication structure that establishes a new link between two inside players if they can communicate via outside players. Thus, it is shown that, if communication structures are present, the core may still be characterized on balanced TU games, on totally balanced TU games, on NTU games with a nonempty core, on the domains of all TU or NTU games, and on several other interesting domains of TU and NTU games. As a byproduct we construct, for any NTU game with communication structure, a certain classical NTU game with the same core that may be regarded as its Myerson restricted NTU game.
\end{abstract}

Keywords: TU and NTU game · Solution · Communication structure · Core

JEL Classification: C71

\section{Introduction}

A cooperative transferable utility (TU) game models a situation in which each coalition $S$ in a finite set $N$ of players can realize a certain joint profit, the worth $v(S)$ of the coalition, if it forms. The core applied to such a game is the set of all proposals of how to share $v(N)$ such that each coalition $S$ receives at least $v(S)$. In a more general model of TU games, Faigle (1989) has investigated the cores of games with restricted cooperation, i.e., when the coalition function is restricted to a set of feasible coalitions. In the present paper we consider the kind of restriction introduced by Myerson (1977) that requires that only connected coalitions can form. A coalition is connected if its members are able to communicate with each other using communication links inside the coalition of a given simple graph. The grand coalition $N$ is here replaced by the natural coalition structure of connected components. For such games with communication structures Myerson introduced the Myerson value, namely the Shapley value of the corresponding classical so-called "Myerson restricted" game. Two solutions related to the core, namely versions of Schmeidler's (1969) nucleolus and of Davis and Maschler's (1965) kernel have

*This research was supported by the Spanish Ministerio de Ciencia e Innovación under project ECO2012-33618, cofunded by the ERDF. Moreover, the second author was supported by The Danish Council for Independent Research|Social Sciences under the FINQ project.

${ }^{\dagger}$ Faculty of Economics and Business Administration, Basque Country University, Lehendakari Aguirre 83, 48015 Bilbao, Spain. E-mail: mj.albizuri@ehu.es

${ }^{\ddagger}$ Department of Business and Economics and COHERE, University of Southern Denmark, Campusvej 55, 5230 Odense M, Denmark. E-mail: psu@sam.sdu.dk 
been characterized (Khmelnitskaya and Sudhölter 2013), and also the core has been investigated. For example, Herings, van der Laan, Talman, and Yang (2010) checked if the core is nonempty for a subclass of games, and they discussed when their solution lies in the core. Hence, the core has been considered as a "reference solution" and, as far as we know, until now no attempts have been made to support the core itself as a solution for games with communication structures. Our analysis of the core, by contrast, emphasizes the axiomatic approach, and we show that some of the well-known characterizations of the core of TU games also hold when communication structures are present.

We generalize some axiomatizations of the core also to nontransferable utility (NTU) games with communication structures. The main employed axioms, both in the TU and NTU cases, are the converse reduced game property and versions of the reduced game property with respect to a reduced game à la Davis and Maschler (1965), the definition of which requires to additionally define the reduced communication structure. A new link between two players of $S$ in the reduced communication structure (Khmelnitskaya and Sudhölter 2013) on $S$ is established if these players can communicate via some players that do not belong to $S$. Now, RGP, the reduced game property (Peleg 1985, 1986), basically requires that the restriction $x_{S}$ to the coalition $S \subseteq N$ of a proposal $x_{N}$ in the solution applied to the game on $N$ be in the solution of the reduced game on $S$, whereas RCP, the reconfirmation property (Hwang and Sudhölter 2001), basically (a slightly weaker property will be employed) requires that for any element $y_{S}$ of the solution to the reduced game on $S$, its combination with the remaining coordinates of $x,\left(y_{S}, x_{N \backslash S}\right)$ belong to the solution to the game on $N$ as well. Hence, for one-point solutions, RGP and RCP coincide, and this property was first used by Sobolev (1975). Finally CRGP, the converse RGP (Peleg 1985, 1986), requires that a Pareto optimal proposal belong to the solution of a game if its restriction to any pair of players belongs to the solution of the corresponding reduced game of these two players. We show that the following characterizations of the core provided by Peleg also hold for games with communication structures. The core is characterized by

(1) non-emptiness (NE), individual rationality (IR), RGP, and superadditivity (SUPA) on balanced TU games;

(2) NE, IR, the weak (W) RGP, CRGP, and SUPA on totally balanced TU games;

(3) NE, IR, WRGP, and CRGP on NTU games with nonempty cores;

(4) NE, IR, and RGP on NTU games with nonempty cores.

Moreover, we show that the following axiomatizations (Hwang and Sudhölter 2001) by

(5) the zero-inessential game property (ZIG), anonymity, covariance under strategic equivalence (COV), WRGP, RCP, CRGP, and reasonableness from below (REAS) on TU or NTU games;

(6) ZIG, COV, WRGP, RCP, CRGP, and REAS on many classes of TU and NTU games

can be generalized. For TU games, REAS may even be replaced by the weaker requirement that the solution to any game be bounded.

Other axiomatizations of the core can be found in the literature. We mention only two of them. Tadenuma (1992) characterizes the core for TU and NTU games by NE, IR, and a reduced game property which 
refers to a reduced game that has not been generalized to games with communication structures. Due to our focus on the aforementioned versions of the reduced game property, we also did not generalize the approach of Serrano and Volij (1998), who employ the requirement that, if the restriction of an individually rational Pareto optimal proposal to any proper subcoalition belongs to the solution of the corresponding reduced game, then the entire proposal belongs to the solution of the original game.

The first part of the paper presents the results on TU games, namely first the generalizations of the mentioned statements (5) and (6), secondly of (1), and finally of (2), while the second part deals with the NTU case, i.e., statements (3), (4), and then (5) and (6). In more detail the paper is organized as follows. In Sect. 2 basic definitions of the reduced communication structure and of possible intuitive properties of solutions for the TU case are recalled, some notation is provided, and it is remarked that the core of a TU game with communication structure coincides with the core of the corresponding classical Myerson restricted TU game. Sect. 3 is devoted to generalize the aforementioned axiomatizations (5) and (6) to TU games with communication structures, while Sect. 4 is devoted to the aforementioned generalizations of (1) and (2). It is shown that (1) is no longer valid for communication structures unless there exists an infinite universe of potential players, whereas classically a universe of three players was already sufficient. Sect. 5 first introduces some notation on NTU games and presents the definition of the Myerson restricted NTU game of an NTU game with communication structure with the following properties: The Myerson restricted NTU game generalizes the Myerson restricted TU game, and the core of the Myerson restricted NTU game coincides with the core of the NTU game with communication structure. Hence, one may define balanced and totally balanced NTU games with communication structures. Moreover, this section has two subsections, the first dealing with the characterizations (3) and (4), the second with (5) and (6) on NTU games. In Sect. 6 it is shown that each axiom in each of the characterizations is logically independent of the remaining axioms. Finally, in Sect. 7 we remark that the intersection of the prekernel and the core on balanced TU games with communication structures is characterized similarly to the classical case, that our employed CRGP may be replaced by a weaker version, and that all results may be extended to TU and NTU games with conference structures à la Myerson (1980).

\section{Some notation, definitions, and preliminaries}

Let $U$ be a set, the universe of players, let $\mathcal{F}$ denote the set of all coalitions, i.e., finite nonempty subsets of $U$, and, for $N \in \mathcal{F}$, let $\mathcal{F}^{N}$ denote the set of subcoalitions of $N$, i.e., $\mathcal{F}^{N}=\{S \subseteq N \mid S \neq \emptyset\}$. A (cooperative) transferable utility (TU) game with communication structure is a triple $(N, v, g)$ such that $N$ is a coalition, $v: 2^{N} \rightarrow \mathbb{R}, v(\emptyset)=0$ (i.e., $(N, v)$ is a $\mathrm{TU}$ game), and $(N, g)$ is a simple graph (i.e., $g$ is a set of 2-element subsets of the vertex set $N)$, called communication structure. Let $(N, g)$ be a communication structure, $(N, v)$ be a TU game, $S \in \mathcal{F}^{N}$, and $i, j \in S$. Then the vertices $i$ and $j$ are connected in $S$ by $g$ if $i=j$ or there exist vertices $i=k_{1}, \ldots, k_{\ell}=j \in S$ such that $\left\{k_{t}, k_{t+1}\right\} \in g$ for all $t=1, \ldots, \ell-1$. Let $S / g=\{\{i \in S \mid i$ and $j$ are connected in $S$ by $g\} \mid j \in S\}$ denote the set of components of $S$ with respect to (w.r.t.) $g$ and say that $S$ is connected by $g$ if $|S / g|=1$. Here and in the sequel $|D|$ denotes the cardinality of $D$ if $D$ is a set. Let $\mathcal{F}_{g}^{N}$ denote the set of connected coalitions of $N$ w.r.t. $g$. A TU game with communication structure $(N, v, g)$ models a situation in which only the connected coalitions may form. Hence, the worth $v(S)$ of $S \in \mathcal{F}^{N} \backslash \mathcal{F}_{g}^{N}$ is regarded as virtual only. In the case that $(N, g)$ is the complete graph, i.e., $\mathcal{F}_{g}^{N}=\mathcal{F}^{N},(N, v, g)$ is identified with the (classical) TU 
game $(N, v)$. Let

$$
\begin{aligned}
X^{*}(N, v, N / g) & =\left\{x \in \mathbb{R}^{N} \mid x(R) \leqslant v(R) \text { for all } R \in N / g\right\} \text { and } \\
X(N, v, N / g) & =\left\{x \in \mathbb{R}^{N} \mid x(R)=v(R) \text { for all } R \in N / g\right\}
\end{aligned}
$$

denote the set of feasible payoffs and Pareto optimal (also called component efficient in the literature) feasible payoffs (preimputations is synonymous), respectively, where $x(S)=\sum_{i \in S} x_{i}(x(\emptyset)=0)$ for $S \subseteq N$ and $x \in \mathbb{R}^{N}$. We also denote by $x_{S}=\left(x_{i}\right)_{i \in S} \in \mathbb{R}^{S}$ the restriction of $x$ to $S$ and write $x=\left(x_{S}, x_{N \backslash S}\right)$.

Hence, the core of $(N, v, g)$, denoted by $C(N, v, g)$, is defined by

$$
C(N, v, g)=\left\{x \in X^{*}(N, v, N / g) \mid x(S) \geqslant v(S) \text { for all } S \in \mathcal{F}_{g}^{N}\right\}
$$

so that $C(N, v, g) \subseteq X(N, v, g)$.

Recall that the Myerson restricted (classical) TU game $(N, v / g)$ is defined by

$$
(v / g)(S)=\sum_{T \in S / g} v(T) \text { for all } S \subseteq N
$$

Thus,

$$
C(N, v, g)=C(N, v / g)
$$

We now recall (Khmelnitskaya and Sudhölter 2013, Definition 2.4) that the reduced game à la Davis and Maschler (1965) with communication structure of $(N, v, g)$ w.r.t. $S \in \mathcal{F}^{N}$ and $x \in X^{*}(N, v, g)$ is denoted by $\left(S, v_{g}^{S, x}, g^{S}\right)$, where the reduced communication structure $\left(S, g^{S}\right)$ is defined by

$$
g^{S}=\{\{i, j\} \subseteq S \mid i \neq j \text { and } i \text { and } j \text { are connected in }\{i, j\} \cup(N \backslash S) \text { by } g\}
$$

and the reduced coalition function $v_{g}^{S, x}$ is defined by

$$
v_{g}^{S, x}(T)= \begin{cases}v(R)-x(R \backslash T), & \text { if } T=S \cap R \text { for some } R \in N / g, \\ \max \left\{v(T \cup Q)-x(Q) \mid Q \subseteq N \backslash S, T \cup Q \in \mathcal{F}_{g}^{N}\right\}, & \text { if } T \in \mathcal{F}_{g^{S}}^{S} \text { and } T \notin S / g^{S}, \\ \max \{v(T \cup Q)-x(Q) \mid Q \subseteq N \backslash S\}, & \text { if } T \in \mathcal{F}^{S} \backslash \mathcal{F}_{g^{S}}^{S} .\end{cases}
$$

Note that

- $v_{g}^{S, x}$ coincides with the coalition function, denoted $v^{S, x}$, of the classical reduced TU game if $(N, g)$ is complete - the first row of (2.3) only applies to $R=S$, in the second row " $T \cup Q \in \mathcal{F}_{g}^{N}$ " and " $T \in \mathcal{F}_{g^{S}}^{S}$ " are vacuously satisfied so that the case of the third row does not occur;

- $v_{g}^{S, x}$ is well-defined because $\mathcal{F}_{g^{S}}^{S}=\left\{T \cap S \mid T \in \mathcal{F}_{g}^{N}\right\} \backslash\{\emptyset\}$;

- we adopted the classical definition of Davis and Maschler for the worth if the coalition $T$ is not connected, i.e., $T \in \mathcal{F}^{S} \backslash \mathcal{F}_{g^{S}}^{S}$ (see the third row of (2.3)), because there is no need to change the definition of a virtual worth.

Note that reducing and Myerson restricting may not commute even for core elements in the following sense: If $x \in C(N, v, g)=C(N, v / g)$ and $S \in \mathcal{F}^{N}$, then the reduced coalition function of the Myerson restricted TU game $(v / g)^{S, x}$ may differ from the coalition function of the Myerson restricted reduced 
TU game $\left(v_{g}^{S, x}\right) / g^{S}$. Indeed, if $|N| \geqslant 4, g$ consists of all but one link $\{k, \ell\}$, and if $v(S)=0$ for all but one coalition $T=\{j, k, \ell\}$ of cardinality 3, whereas $v(T)=-1$, then $x=0 \in \mathbb{R}^{N}$ is the unique core element and, with $S=N \backslash\{j\},\left(S, g^{S}\right)$ is complete and $v_{g}^{S, x}(\{k, \ell\})=v(T)-x_{j}=-1$, whereas $(v / g)^{S, x}(\{k, \ell\})=\max \left\{v(\{k\})+v(\{\ell\}), v(T)-x_{j}\right\}=0$.

Let $\Gamma_{U}$ and $\Gamma_{U \text {,class }}$ denote the set of $\mathrm{TU}$ games with communication structures and the subset of (classical) TU games, respectively. A solution is a mapping $\sigma$ that assigns a subset $\sigma(N, v, g)$ of $X^{*}(N, v, g)$ to any TU game with communication structure $(N, v, g)$. Its restriction to a set $\Gamma \subseteq \Gamma_{U}$ is again denoted by $\sigma$. Moreover, a solution on $\Gamma$ is the restriction to $\Gamma$ of some solution. A solution $\sigma$ on $\Gamma \subseteq \Gamma_{U}$ satisfies

- nonemptiness (NE) if $\sigma(N, v, g) \neq \emptyset$ for all $(N, v, g) \in \Gamma$;

- the zero inessential 2-person game property (ZIG) if for every $(N, v, g) \in \Gamma$ such that $|N|=2$ and $v(S)=0$ for all $S \subseteq N, \sigma(N, v, g) \neq \emptyset$;

- boundedness (BOUND) if $\sigma(N, v, g)$ is a bounded set for all $(N, v, g) \in \Gamma$;

- reasonableness from below (REAS) if for all $(N, v, g) \in \Gamma$ and $x \in \sigma(N, v, g)$,

$$
x_{i} \geqslant \min \left\{v(S \cup\{i\})-v(S) \mid S \subseteq N \backslash\{i\}, S, S \cup\{i\} \in \mathcal{F}_{g}^{N} \cup\{\emptyset\}\right\} \text { for all } i \in N \text {; }
$$

- Pareto optimality (PO) if $\sigma(N, v, g) \subseteq X(N, v, N / g)$ for all $(N, v, g) \in \Gamma$;

- anonymity (AN) if the following condition is satisfied: If $(N, v, g) \in \Gamma, \pi: N \rightarrow U$ is an injection, and if $(\pi(N), \pi v, \pi g) \in \Gamma$, then $\sigma(\pi(N), \pi v, \pi g)=\pi(\sigma(N, v, g))$, where $(\pi v)(\pi(S))=v(S)$ for all $S \subseteq N, \pi g=\{\{\pi(i), \pi(j)\} \mid\{i, j\} \in g\}$, and $\pi(x)=y \in \mathbb{R}^{\pi(N)}$ is defined by $y_{\pi(i)}=x_{i}$ for all $x \in \mathbb{R}^{N}$ and all $i \in N$;

- covariance under strategic equivalence (COV) if the following condition is satisfied for all $(N, v, g)$ and all $(N, w, g) \in \Gamma$ : If $\alpha>0, \beta \in \mathbb{R}^{N}$, and $w=\alpha v+\beta$, then $\sigma(N, w, g)=\alpha \sigma(N, v, g)+\beta$;

- the reduced game property (RGP) if for any $(N, v, g) \in \Gamma$ and any $x \in \sigma(N, v, g)$ the following condition holds: If $S \in \mathcal{F}^{N}$, then $\left(S, v_{g}^{S, x}, g^{S}\right) \in \Gamma$ and $x_{S} \in \sigma\left(S, v_{g}^{S, x}, g^{S}\right)$;

- the converse reduced game property (CRGP) if the following condition holds for any $(N, v, g) \in \Gamma$ with $|N|-|N / g| \geqslant 1$ : If $x \in X(N, v, N / g)$, if $\left(S, v_{g}^{S, x}, g^{S}\right) \in \Gamma$ and $x_{S} \in \sigma\left(S, v_{g}^{S, x}, g^{S}\right)$ for all $S \in\{\{k, \ell\} \subseteq N \mid k \neq \ell$ and $k, \ell \in R$ for some $R \in N / g\}$, then $x \in \sigma(N, v, g)$;

- the reconfirmation property (RCP) if the following condition is satisfied for every $(N, v, g) \in \Gamma, x \in$ $\sigma(N, v, g)$, and $S \in \mathcal{F}^{N}$ : If $\left(S, v_{g}^{S, x}, g^{S}\right) \in \Gamma, y \in \sigma\left(S, v_{g}^{S, x}, g^{S}\right)$, and $y(R) \geqslant x(R)$ for all $R \in S / g^{S}$, then $\left(y, x_{N \backslash S}\right) \in \sigma(N, v, g)$;

- individual rationality (IR) if for any $(N, v, g) \in \Gamma$ and $x \in \sigma(N, v, g), x_{i} \geqslant v(\{i\})$ for all $i \in N$;

- superadditivity (SUPA) if for any $(N, v, g),(N, w, g) \in \Gamma$ the following condition holds: If $(N, v+$ $w, g) \in \Gamma$, then $\sigma(N, v, g)+\sigma(N, w, g) \subseteq \sigma(N, v+w, g)$.

Recall that the definition of the weak reduced game property (WRGP) differs from that of RGP only inasmuch as $|S| \leqslant 2$ is additionally required. Moreover, note that with the condition $y(R) \geqslant x(R)$ for all $R \in S / g^{S}$ our RCP is a generalization of a property that Hwang and Sudhölter (2001) called weak RCP 
(see also Remark $5.5(1)$ ). For interpretations of and further references to the foregoing properties in the case that $\Gamma$ is a set of games, i.e., if $(N, g)$ is the complete graph for each $(N, v, g) \in \Gamma$, see Peleg (1986) and Hwang and Sudhölter (2001). Finally, note that RGP and CRGP are used in characterizations of the prenucleolus and prekernel on $\Gamma_{U}$ (Khmelnitskaya and Sudhölter 2013).

\section{The core on various sets of TU games}

This section is devoted to generalize the characterizations of Hwang and Sudhölter (2001) of the core on various sets of TU games to TU games with communication structures. The main result is the following theorem.

Theorem 3.1 The core is the unique solution on $\Gamma_{U}$ that satisfies $\mathrm{ZIG}, \mathrm{AN}, \mathrm{COV}$, WRGP, RCP, CRGP, and BOUND, provided that $|U| \geqslant 5$.

We postpone the proof of the foregoing theorem and first present the following remark.

Remark 3.2 The core satisfies ZIG, AN, COV, RCP, CRGP, IR, SUPA, and BOUND on any subset of $\Gamma_{U}$. Moreover, on subsets of $\Gamma_{U}$ that contain with each TU game and core element all (2-person) reduced TU games, the core satisfies (W)RGP.

Indeed, if $(N, v, g) \in \Gamma_{U}$ satisfies $v(S)=0$ for all $S \subseteq N$, then $C(N, v, g)$ consists of the single element $0 \in \mathbb{R}^{N}$. This observation shows ZIG. Moreover, as its definition does not depend on the names of the players, the core satisfies AN. It is straightforward to verify that the core satisfies COV. RGP, RCP, SUPA, and CRGP may be verified precisely as in the case of ordinary TU games. By definition the core is individually rational. Finally, as all singletons $\{i\}, i \in N$, of any $(N, v, g) \in \Gamma_{U}$ are connected coalitions, $C(N, v, g)$ is bounded.

When $\Gamma_{U}$ is replaced by $\Gamma_{U \text {,class }}$, then the corresponding version of the foregoing theorem is proved by Hwang and Sudhölter (2001, Theorem 4.2). The current generalization is an immediate consequence of the following lemma and remark. Let $\Gamma \subseteq \Gamma_{U}$ and let $\sigma, \sigma^{1}$, and $\sigma^{2}$ be solutions on $\Gamma$.

Remark 3.3 (1) If $\sigma^{1}$ satisfies PO and WRGP and $\sigma^{2}$ satisfies CRGP and if $\sigma^{1}(N, v, g) \subseteq \sigma^{2}(N, v, g)$ for every $(N, v, g) \in \Gamma$ with $|N| \leqslant 2$, then $\sigma^{1}$ is a subsolution of $\sigma^{2}$, i.e., $\sigma^{1}(M, w, h) \subseteq \sigma^{2}(M, w, h)$ for all $(M, w, h) \in \Gamma$.

(2) If $\sigma$ satisfies COV, WRGP, and BOUND, then $\sigma$ satisfies PO.

Indeed, this remark may be shown by suitably rewriting the proofs of Remark 3.7 and Lemma 4.3 of Hwang and Sudhölter (2001).

We now generalize the expression "subgame" to games with communication structures: Let $(N, v, g) \in \Gamma_{U}$ and $S \in \mathcal{F}^{N}$. The subgame on $S,\left(S, v^{\prime}, g^{\prime}\right)$, is defined by $v^{\prime}(T)=v(T)$ for all $T \subseteq S$ (i.e., $\left(S, v^{\prime}\right)$ is the classical subgame of $(N, v)$ where the coalition function $v^{\prime}$ is the restricted to $v$ to the subsets of $\left.S\right)$ and $g^{\prime}=\{L \in g \mid L \subseteq S\}$ (i.e., $\left(S, g^{\prime}\right)$ is the induced subgraph on $S$ of $(N, g)$ ). By slightly abusing notation we denote this subgame by $(S, v, g)$. Moreover, note that the Myerson restricted game of the subgame 
$(S, v, g)$ is the subgame on $S$ of the Myerson restricted game $(N, v / g)$, hence this TU game is denoted by $(S, v / g)$.

With this notation, $(N, v, g)$ is called (totally) balanced if $(N, v / g)$ is (totally) balanced. According to Bondareva (1963) and Shapley (1967), $(N, v, g)$ is (totally) balanced if and only if $C(N, v, g) \neq \emptyset$ $\left(C(S, v, g) \neq \emptyset\right.$ for all $\left.S \in \mathcal{F}^{N}\right)$. Let $\Gamma_{U}^{t b}$ denote the set of totally balanced TU games with communication structures.

Lemma 3.4 Assume that $|U| \geqslant 3, \Gamma_{U}^{t b} \subseteq \Gamma$, and that $\sigma$ satisfies ZIG, COV, WRGP, CRGP, and BOUND. If $\sigma$ coincides with $C$ on the set of all 2-person classical games in $\Gamma \cap \Gamma_{U \text {,class, }}$, then $\sigma$ coincides with $C$ for each 2-person game in $\Gamma$.

Proof: Let $(N, v, g) \in \Gamma \backslash \Gamma_{U \text {,class }}$ be a 2-person game. Then $N \notin g$. We may assume that $M=\{1,2,3\} \subseteq$ $U$ and $N=\{1,2\}$. Define $x \in \mathbb{R}^{N}$ by $x_{i}=v(\{i\})$ for $i \in N$. As $X(N, v, g)=C(N, v, g)=\{x\}$, by Remark $3.3(2)$ it remains to show $\sigma(N, v, g) \neq \emptyset$. Let $(M, h)$ be the graph given by $h=\{\{2,3\}\}$ and let $(M, w, h) \in \Gamma_{U}$ be defined by $w(T)=v(T \cap N)$ for all $T \subseteq M$. Then $y=(x, 0) \in C(M, w, h)$. Let $S=\{2,3\}$. As $\left(S, h^{S}\right)$ is complete and $y_{S} \in C\left(S, w_{h}^{S, y}\right)=\sigma\left(S, w_{h}^{S, y}\right), y \in \sigma(M, w, h)$ by CRGP. By WRGP, $y_{N}=x \in \sigma\left(N, w_{h}^{N, y}, h^{N}\right)$ and $\left(N, w_{h}^{N, y}, h^{N}\right)=(N, v, g)$, the proof is complete.

Now, we are able to prove our theorem.

Proof of Theorem 3.1: By Remark 3.2 it remains to prove the uniqueness part. Let $\sigma$ satisfy the desired properties. By Theorem 4.2 of Hwang and Sudhölter (2001), $\sigma$ coincides with the core for each classical TU game. By Lemma $3.4 \sigma$ coincides with $C$ for any game $(N, v, g)$ with communication structures that satisfies $|N| \leqslant 2$. By Remark $3.3(2), \sigma$ satisfies PO. By Remark 3.3 (1) alternatingly applied to $\sigma^{i}=\sigma$ and $\sigma^{3-i}=C$ for $i=1,2$ shows that $\sigma$ is a subsolution of $C$ and, vice versa, $C$ is a subsolution of $\sigma$.

We now show that similarly to the case of classical TU games, the statement of Theorem 3.1 is still valid for many interesting subsets of $\Gamma_{U}$.

Theorem 3.5 If $\Gamma_{U}^{t b} \subseteq \Gamma \subseteq \Gamma_{U}$ such that any $(N, v, g) \in \Gamma$ with $|N|=2$ is balanced, then the core on $\Gamma$ is the unique solution that satisfies ZIG, COV, WRGP, RCP, CRGP, and BOUND, provided that $|U| \geqslant 5$.

Proof: Again just the uniqueness part has to be proved. Let $\sigma$ be a solution that satisfies the desired properties and let $(N, v, g) \in \Gamma$. If $(N, g)$ is complete, then $\sigma(N, v)=C(N, v)$ by Theorem 5.1 of Hwang and Sudhölter (2001). By consecutively applying Lemma 3.4, Remark 3.3 (2), and twice Remark 3.3 (1) the proof may be finished along the corresponding part of the proof of Theorem 3.1.

\section{The core on balanced and totally balanced TU games}

We now show that Peleg's (1986) characterization of the core on balanced TU games by NE, IR, RGP, and SUPA may be generalized to TU games with communication structures, provided that $|U|=\infty$. For this purpose denote by $\Gamma_{U}^{b}$ the set of all balanced TU games with communication structures.

For completeness reasons we present the proof of the following lemma that is similar to the proof of Peleg's (1986) Lemma 5.6. 
Lemma 4.1 If a solution $\sigma$ on $\Gamma \subseteq \Gamma_{U}$ satisfies IR and WRGP, then it is a subsolution of the core.

Proof: Let $(N, v, g) \in \Gamma$ and $x \in \sigma(N, v, g)$. We first show PO, i.e., $x \in X(N, v, N / g)$. Assume, on the contrary, that $x(R)<v(R)$ for some $R \in N / g$. Let $i \in R$, then $x_{i}<v(R)-x(R \backslash\{i\})=v_{g}^{\{i\}, x}(\{i\})$ so that a contradiction to IR and WRGP has been obtained.

Let $n=|N|$. If $n \leqslant 2$, PO and IR imply that $x \in C(N, v, g)$. If $n>2$ and $x(S)<v(S)$ for some $S \in \mathcal{F}_{g}^{N}$, then let $R \in N / g$ such that $S \subseteq R$. By PO, $S \neq R$ so that there exists $j \in R \backslash S$. As $S \neq \emptyset$, there exists $i \in S$. Then $x_{i}<v_{g}^{\{i, j\}, x}(\{i\})$ so that WRGP and IR yield the desired contradiction.

Theorem 4.2 The core is the unique solution on $\Gamma_{U}^{b}$ that satisfies NE, IR, RGP, and SUPA, provided that $|U|=\infty$.

In the proof of this theorem and in the definition of the Myerson restricted NTU game (see (5.6)) let, for $T \subseteq M \in \mathcal{F}, \chi^{T, M} \in \mathbb{R}^{M}$ denote the indicator vector of $T$ w.r.t. $M$ defined by

$$
\chi_{i}^{T, M}=\left\{\begin{array}{l}
1, \text { if } i \in T, \\
0, \text { if } i \in M \backslash T .
\end{array}\right.
$$

If the ground set $M=N$ is clear from the context, then we sometimes omit the second superscript $M$, i.e., use the notation $\chi^{T}=\chi^{T, N}$.

Proof: By definition of $\Gamma_{U}^{b}$ the core satisfies NE so that by Remark 3.2 only the uniqueness part has to be shown. Let $\sigma$ be a solution on $\Gamma_{U}^{b}$ that satisfies the desired properties and let $(N, v, g) \in \Gamma_{U}^{b}$. Denote $n=|N|$. By Lemma 4.1 it remains to show that $C(N, v, g) \subseteq \sigma(N, v, g)$. Let $x \in C(N, v, g)$. Recall that $T \in \mathcal{F}^{N}$ is a clique of $(N, g)$ if the induced subgraph on $T$ is complete. We first consider the case that $N$ is the disjoint union of cliques that have cardinalities of at least 3, i.e., there exists a partition $\mathcal{T}$ of $N$ such that $|T| \geqslant 3$ and the induced subgraph $(T, g)$ is complete for all $T \in \mathcal{T}$. Define $(N, u, g)$ by $u(\{i\})=v(\{i\})$ for all $i \in N$ and $u(S)=x(S)$ for all other coalitions $S \subseteq N$. Then $x \in C(N, u, g)$. Moreover, for any $S \subseteq N$ with $|S| \geqslant 2, \sum_{i \in S} \frac{\chi^{S \backslash\{i\}}}{|S|-1}=\chi^{S}$. As $N=\bigcup_{T \in \mathcal{T}} T$ and $T \cap T^{\prime}=\emptyset$ for all $T, T^{\prime} \in \mathcal{T}$ with $T \neq T^{\prime}$, we have

$$
\sum\left\{\sum_{i \in T} \frac{\chi^{T \backslash\{i\}}}{|T|-1} \mid T \in \mathcal{T}\right\}=\chi^{N},
$$

i.e., $\{T \backslash\{i\} \mid T \in \mathcal{T}\}$ is a balanced collection of connected coalitions. Moreover, the vectors $\chi^{T \backslash\{i\}}$ where $T \in \mathcal{T}$ and $i \in T$ span $\mathbb{R}^{N}$ - in fact these vectors form a vector space basis of $\mathbb{R}^{N}$. Therefore, $C(N, u, g)=\{x\}$. By NE, $x \in \sigma(N, u, g)$. As $(v-u)(\{i\})=(v-u)(R)=0$ for all $i \in N$ and $R \in N / g$ and $(v-u)(S) \leqslant 0$ for all $S \in \mathcal{F}_{g}^{N}, C(N, v-u, g)=\{0\}$. By NE, $0 \in \sigma(N, v-u, g)$ and, by SUPA, $x=x+0 \in \sigma(N, u+(v-u), g)$ so that the proof is finished in this case.

Now, we can complete the proof. In the general case, by assumption there exists $M \subseteq U$ such that $N \subseteq M$ and $|M|=3 n$. For each $i \in N$ choose $i^{\prime}, i^{\prime \prime} \in M$ such that $M$ is the disjoint union of $N$, $N^{\prime}=\left\{i^{\prime} \mid i \in N\right\}$, and $N^{\prime \prime}=\left\{i^{\prime \prime} \mid i \in N\right\}$. Define the graph $(M, h)$ by

$$
h=g \cup\left\{\left\{i, i^{\prime}\right\} \mid i \in N\right\} \cup\left\{\left\{i, i^{\prime \prime}\right\} \mid i \in N\right\} \cup\left\{\left\{i^{\prime}, i^{\prime \prime}\right\} \mid i \in N\right\} .
$$

Hence, all of the sets $\left\{i, i^{\prime}, i^{\prime \prime}\right\}, i \in N$, are cliques of $(M, h)$ of cardinality 3 that form a partition of $M$. Moreover, let the TU game $(N, w)$ be defined by $w(S)=v(S \cap N)$, i.e., all players in $M \backslash N$ are 
null-players. Then, for $S \subseteq M$, if $S \in \mathcal{F}_{h}^{M}$, then $S \cap N \in \mathcal{F}_{g}^{N}$. Let $y \in \mathbb{R}^{M}$ be determined by $y_{N}=x$ and $y_{M \backslash N}=0 \in \mathbb{R}^{M \backslash N}$. Then $y \in C(M, w, h)$, hence $y \in \sigma(M, w, h)$ by the former case. As $v=w_{h}^{N, y}$, $h^{N}=g$, and $y_{N}=x$, RGP finishes the proof.

Theorem 4.2 generalizes Peleg's (1986) Theorem 5.4. However, for classical TU games $|U|=\infty$ may be replaced by $|U| \geqslant 3$ and RGP may be replaced by WRGP. We now show by means of the following two examples that without the assumption $|U|=\infty$ our theorem is not valid and that WRGP does not replace $R G P$.

Example 4.3 Recall that a solution $\sigma$ on $\Gamma \subseteq \Gamma_{U}$ is a standard solution if, for any $(\{i, j\}, w) \in \Gamma$ such that $i \neq j$, with $y \in \mathbb{R}^{\{i, j\}}$ defined by $y_{i}=\frac{w(\{i\})-w(\{j\})+w(N)}{2}, \sigma(\{i, j\}, w)=\{y\}$. Thus, $y \in C(\{i, j\}, w)$ if $(\{i, j\}, w)$ is balanced. Now, for $(N, v, g) \in \Gamma_{U}$, define $\widehat{\sigma}$ as follows: If $N=U$, then $x \in \widehat{\sigma}(N, v, g)$ if and only if for any $S \in N / g$ with $|S|=2,\left\{x_{S}\right\}$ is the standard solution for the subgame $(S, v, g)$. If $N \varsubsetneqq U$, then $\widehat{\sigma}(N, v, g)=C(N, v, g)$. On $\Gamma_{U}^{b}$ our solution $\widehat{\sigma}$ satisfies $\mathrm{NE}$ because the reduced game w.r.t. any connected component with a complete induced subgraph coincides with the corresponding subgame and the core satisfies RCP. As the standard solution is additive, $\widehat{\sigma}$ satisfies SUPA. As a subsolution of the core it satisfies IR. As it coincides with the core whenever not all players are present, it satisfies RGP. Finally, if $2 \leqslant|U|<\infty$, then $\widehat{\sigma} \neq C$.

We now present an example of a solution on $\Gamma_{U}^{b}$ that satisfies NE, IR, WRGP, and SUPA, but violates RGP if $|U| \geqslant 3$.

Example 4.4 Recall that $i \in N$ is a leaf vertex of the graph $(N, g)$ if $i$ has a unique adjacent vertex, i.e., if the degree of $i$ is 1 . Let $L(g)$ be the set of leaf vertices whose adjacent vertices have at least degree 2. For any $(N, v, g) \in \Gamma_{U}^{b}$ define $\widetilde{\sigma}(N, v, g)=\left\{x \in C(N, v, g) \mid x_{i}=v(\{i\})\right.$ for all $\left.i \in L(g)\right\}$. If a graph $(N, g)$ has at most two vertices, then $L(g)=\emptyset$. Hence, $\widetilde{\sigma}$ coincides with the core for TU games with at most 2 players so that $\widetilde{\sigma}$ satisfies IR and WRGP. It is straightforward to verify that it also satisfies SUPA. Moreover, if $y \in C(N, v, g)$ and $x \in \mathbb{R}^{N}$ is defined, for $i \in N$, by

$$
x_{i}= \begin{cases}v(\{i\}) & , \text { if } i \in L(g), \\ y_{i}+y_{j}-v(\{j\}) & , \text { if } i \text { if adjacent to some (unique) } j \in L(g), \\ y_{i} & , \text { otherwise, }\end{cases}
$$

then $x \in \widetilde{\sigma}(N, v, g)$ so that $\widetilde{\sigma}$ satisfies NE. Finally, if $|U| \geqslant 3$, say $N=\{1,2,3\} \in U$, then let $(N, v)$ be the unanimity game, i.e., the game defined by $v(N)=1$ and $v(S)=0$ for all $S \varsubsetneqq N$, and $(N, g)$ be the graph defined by $g=\{\{1,2\},\{2,3\}\}$, then $(0,1,0)$ is the unique element of $\widetilde{\sigma}(N, v, g)$ whereas $C(N, v, g)$ is the entire unit simplex in $\mathbb{R}^{N}$. Hence, $\widetilde{\sigma} \neq C$ so that $\widetilde{\sigma}$ must violate RGP.

As stated by Peleg (1989) and proved by Sudhölter and Peleg (2002, Theorem 3), the core on totally balanced TU games is characterized by NE, IR, WRGP, CRGP, and SUPA, provided that $|U| \geqslant 4$. The following corollary generalizes the foregoing characterization to totally balanced TU games with communication structures.

Corollary 4.5 On $\Gamma_{U}^{t b}$ the core is characterized by NE, IR, WRGP, CRGP, and SUPA, provided that $|U| \geqslant 4$. 
Proof: By Remark 3.2 only the uniqueness part is left. Let $\sigma$ be a solution on $\Gamma_{U}^{t b}$ that satisfies the desired properties and let $(N, v, g) \in \Gamma_{U}^{t b}$. By Lemma 4.1, $\sigma(N, v, g) \subseteq C(N, v, g)$, and, by the aforementioned Theorem 3 of Sudhölter and Peleg (2002), $\sigma(N, v, g)=C(N, v, g)$ whenever $(N, g)$ is a complete graph. If $|N|=2$ and $(N, g)$ is not the complete graph, then $C(N, v, g)$ is a singleton so that $\sigma(N, v, g)=C(N, v, g)$ by NE in this case. Hence, Lemma 3.3 (1) applied to $\sigma^{1}=C$ and $\sigma^{2}=\sigma$ completes the proof.

Remark 4.6 Note that Peleg's (1989) Theorem 7.1 may be generalized as well: Corollary 4.5 remains valid if $\Gamma_{U}^{t b}$ is replaced by an arbitrary set $\Gamma$ of games such that $\Gamma_{U}^{t b} \subseteq \Gamma \subseteq \Gamma_{U}^{b}$.

\section{The NTU case}

Some additional notation is needed. For $N \in \mathcal{F}, x, y \in \mathbb{R}^{N}, x \leqslant y$ if $x_{i} \leqslant y_{i}$ for all $i \in N, x<y$ if $x \leqslant y$ and $x \neq y$, and $x \ll y$ if $x_{i}<y_{i}$ for all $i \in N$. The boundary of a set $X \subseteq \mathbb{R}^{N}$ is denoted by $\partial X$ and we denote $\mathbb{R}_{+}^{N}=\left\{x \in \mathbb{R}^{N} \mid x \geqslant 0\right\}$.

A cooperative nontransferable utility game (NTU game) is a pair $(N, V)$ where $N \in \mathcal{F}$ is finite and $V$ assigns to each $S \in \mathcal{F}^{N}$ a subset $V(S) \subseteq \mathbb{R}^{S}$ such that

$$
\begin{gathered}
V(S) \neq \emptyset, \\
V(S) \text { is closed, }
\end{gathered}
$$

$V(S)$ is comprehensive: $x \in V(S), y \in \mathbb{R}^{S}, y \leqslant x \Rightarrow y \in V(S)$,

$$
V(S) \cap\left(x+\mathbb{R}_{+}^{S}\right) \text { is }{ }^{1} \text { bounded for all } x \in \mathbb{R}^{S},
$$

$$
V(S) \text { is non-leveled: } x, y \in \partial V(S), x \geqslant y \Rightarrow x=y \text {. }
$$

Note that the foregoing definition of an NTU game is customary and may be found in the literature (Peleg 1985).

Let $(N, V, g)$ be an NTU game with communication structure, i.e., $(N, V)$ is an NTU game and $(N, g)$ is a graph. The sets of feasible payoff vectors and of Pareto optimal feasible payoff vectors of $(N, V, g)$ are the sets

$$
\begin{aligned}
& X^{*}(N, V, g)=\left\{x \in \mathbb{R}^{N} \mid x_{R} \in V(R) \text { for all } R \in N / g\right\} \text { and } \\
& X(N, V, g) \quad=\left\{x \in \mathbb{R}^{N} \mid x_{R} \in \partial V(R) \text { for all } R \in N / g\right\},
\end{aligned}
$$

respectively. Let $x \in \mathbb{R}^{N}$ and $S \in \mathcal{F}^{N}$. Then $S$ can improve upon $x$ if there exists $y \in V(S)$ such that $y \gg x_{S}$. The core of $(N, V, g)$, denoted by $C(N, V, g)$, is the set

$$
C(N, V, g)=\left\{x \in X^{*}(N, v, g) \mid \text { No } S \in \mathcal{F}_{g}^{N} \text { can improve upon } x\right\} .
$$

As $V(R), R \in N / g$, is non-leveled, $C(N, V, g) \subseteq X(N, V, g)$. In order to generalize the Myerson restricted game to the NTU context, define with the notation of (4.1), for any $S \in \mathcal{F}^{N}$,

$$
(V / g)(S)=\left\{x+\sum_{T \in S / g} \lambda^{T} \chi^{T, S} \in \mathbb{R}^{S} \mid x_{T} \in V(T), \lambda^{T} \in \mathbb{R} \forall T \in S / g,\left(\sum_{T \in S / g} \lambda^{T} \chi^{T, S}\right)(S)=0\right\} .
$$

Note that the Myerson restricted NTU game $(N, V / g)$ indeed satisfies (5.1) - (5.5) and observe, moreover, that

$$
C(N, V, g)=C(N, V / g)
$$

\footnotetext{
${ }^{1}$ When applied to sets, the "+ denotes the "Minkowski sum", i.e., $x+\mathbb{R}_{+}^{S}=\left\{x+y \mid y \in \mathbb{R}_{+}^{S}\right\}$.
} 
and that, if $(N, V)$ is associated to a TU game $(N, v)$, then $(N, V / g)$ is the NTU game associated with $(N, v / g)$.

Let $S \in \mathcal{F}^{N}$ and $x \in X^{*}(N, V, g)$. Then the reduced object with communication structure w.r.t. $S$ and $x$ of $(N, V, g)$, denoted by $\left(S, V_{g}^{S, x}, g^{S}\right)$, is, for each $T \in \mathcal{F}^{S}$, defined by

$$
V_{g}^{S, x}(T)= \begin{cases}\left\{y \in \mathbb{R}^{T} \mid\left(y, x_{R \backslash S}\right) \in V(R)\right\} & , \text { if } T=S \cap R \text { for some } R \in N / g \\ \bigcup_{Q \in\left\{Q \subseteq N \backslash S \mid T \cup Q \in \mathcal{F}_{g}^{N}\right\}}\left\{y \in \mathbb{R}^{T} \mid\left(y, x_{Q}\right) \in V(T \cup Q)\right\} & , \text { if } T \in \mathcal{F}_{g^{S}}^{S} \backslash\left(S / g^{S}\right) \\ \bigcup_{Q \subseteq N \backslash S}\left\{y \in \mathbb{R}^{T} \mid\left(y, x_{Q}\right) \in V(T \cup Q)\right\} & , \text { otherwise. }\end{cases}
$$

As in the classical case it may easily be deduced that the reduced object $\left(S, V_{g}^{S, x}\right)$ satisfies all properties of an NTU game except (5.1). To be more precise, (5.1) may only be violated for a non-connected subset $T$ of $S$ that is connected w.r.t. $\left(S, g^{S}\right)$. We replace the word "object" by "game" if $\left(S, V_{g}^{S, x}\right)$ is an NTU game, i.e., if $V_{g}^{S, x}(T) \neq \emptyset$ for all $T \in \mathcal{F}^{S}$. Let $\Delta_{U}$ denote the set of all NTU games with communication structures and define

$$
\Delta_{U}^{C}=\left\{(N, V, g) \in \Delta_{U} \mid C(N, V, g) \neq \emptyset\right\} .
$$

In this context a solution $\sigma$ assigns to each $(N, V, g) \in \Delta_{U}$ a subset of $X^{*}(N, V, g)$.

In order to generalize RGP and WRGP to NTU games with communication structures let $\sigma$ be a solution on some $\Delta \subseteq \Delta_{U}$. We say that $\sigma$ satisfies the reduced game property (RGP) if for any $(N, v, g) \in \Gamma$ and any $x \in \sigma(N, v, g)$ the following condition holds: If $S \in \mathcal{F}^{N}$ and if $\left(S, V_{g}^{S, x}\right)$ is an NTU game (i.e., $V_{g}^{S, x}(T) \neq \emptyset$ for all $\left.T \in \mathcal{F}^{S}\right)$, then $\left(S, V_{g}^{S, x}, g^{S}\right) \in \Delta$ and $x_{S} \in \sigma\left(S, V_{g}^{S, x}, g^{S}\right)$. As in the TU context, the definition of the weak reduced game property (WRGP) differs from that of RGP only inasmuch as $|S| \leqslant 2$ is additionally required. However, as $x \in X^{*}(N, V, N / g),\left(S, V_{g}^{S, x}\right)$ is automatically an NTU game because singletons are connected so that the condition "if $\left(S, V_{g}^{S, x}\right)$ is an NTU game" may be deleted in the case $|S| \leqslant 2$.

The generalizations of CRGP, IR $\left(x \in \mathbb{R}^{N}\right.$ is individually rational for $(N, V, g) \in \Delta_{U}$ if $x_{i} \geqslant \max \{V(\{i\})\}$ for all $i \in N), \mathrm{PO}, \mathrm{NE}, \mathrm{AN}$, and BOUND to a set of NTU games with communication structures are straightforward and left to the reader.

Remark 5.1 As for TU games it is straightforward to show the following statements:

(1) The core satisfies CRGP and IR on any subset of $\Delta_{U}$ and that it satisfies RGP or WRGP on subsets that contain all existing reduced games or all reduced games with at most two players, respectively, w.r.t. core elements.

(2) If a solution on a subset of $\Delta_{U}$ satisfies WRGP and IR, then it also satisfies PO.

\subsection{Generalizing Peleg's characterizations}

This subsection is devoted to the generalizations of Peleg's (1985) Theorem 5.5 and Corollary 6.3. Though the subsequent proofs are modifications of his proofs for the case of classical NTU games, all of them are presented in order to keep the presentation self-contained and to allow the interested reader to check inasmuch they differ from the well-known ones. 
Lemma 5.2 If a solution $\sigma$ on some $\Delta \subseteq \Delta_{U}$ satisfies WRGP and IR, then $\sigma(N, V, g) \subseteq C(N, V, g)$ for all $(N, V, g) \in \Delta$.

Proof: By Remark $5.1 \sigma$ satisfies PO. Hence, by IR, $\sigma(N, V, g) \subseteq C(N, V, g)$ whenever $|N| \leqslant 2$. If $|N| \geqslant 3$ and $x \in \sigma(N, V, g)$, then $x_{S} \in \sigma\left(S, V_{g}^{S, x}\right) \subseteq C\left(S, V_{g}^{S, x}\right)$ for all $S \in \mathcal{P}$, where $\mathcal{P}=\{\{k, \ell\} \subseteq N \mid$ $k \neq \ell$ and $k, \ell \in R$ for some $R \in N / g\}$ so that $x \in C(N, V, g)$ by CRGP of $C$.

Lemma 5.3 Let $(N, V, g) \in \Delta_{U}^{C}, \bar{x} \in C(N, V, g)$, and $Q \subseteq U \backslash N$ such that $|Q|=|\{R \in N / g|2 \leqslant| R \mid\}|$, and denote $M=N \cup Q$. Then there exists $(M, W, h) \in \Gamma_{U}^{C}$ such that $C(M, W, h)=\{z\}=\left\{\left(\bar{x}, 0_{M \backslash N}\right)\right\}$ and $\left(N, W_{h}^{N, z}, h^{N}\right)=(N, V, g)$.

Proof: Let $\mathcal{T}=\{R \in N / g|2 \leqslant| R \mid\}$ and select, for any $R \in \mathcal{T}, i(R) \in R$ and $j(R) \in M \backslash N$ such that $M=N \cup\{j(R) \mid R \in \mathcal{T}\}$. Define $h=g \cup\{\{i(R), j(R)\} \mid R \in \mathcal{T}\}$. Let $S \in \mathcal{F}^{M}$. In order to define $W(S)$ we distinguish cases.

(1) If $S \subseteq N$ and $S \notin \mathcal{T}$, then $W(S)=V(S)$.

(2) If $S=R \in \mathcal{T}$, then $W(R)=f(V(R))$ where $f=\left(f_{i}\right)_{i \in R}: \mathbb{R}^{R} \rightarrow \mathbb{R}^{S}$ is defined by

$$
f_{i}(x)=x_{i}+\frac{\left(x_{i}-\bar{x}_{i}\right)_{+}}{1+\left(x_{i}-\bar{x}_{i}\right)_{+}} \text {for all } x \in \mathbb{R}^{R}, i \in S .
$$

As an image of a nonempty set, $W(R) \neq \emptyset$. Moreover,

$$
x_{i} \leqslant f_{i}(x)<x_{i}+1 \text { for all } x \in \mathbb{R}^{R} \text { and } i \in S
$$

implies, together with continuity, (5.2) - (5.4). As $f_{i}$ is strictly monotonic in the sense that $f_{i}(x)<$ $f_{i}(y)$ for all $i \in R$, whenever $x_{i}<y_{i}$, (5.5) is satisfied. As $\bar{x}_{R} \in \partial V(R)$, this strict monotonicity also implies that

$$
\bar{x}_{R} \in \partial W(R)
$$

Moreover, we'll use that

$$
x \in \partial V(R) \backslash\left\{\bar{x}_{R}\right\} \Longrightarrow x \notin \partial W(R) .
$$

Indeed, if $x \in \partial V(R) \backslash\left\{\bar{x}_{R}\right\}$, then there exists $j \in R$ such that $\bar{x}_{j}<x_{j}$. By the definition of $f_{j}$, $f_{j}(x)>x_{j}$, hence $x<f(x) \in W(R)$ so that $x \notin \partial W(R)$ by non-levelness.

(3) If $S \subseteq M \backslash N$, then $W(S)=\left\{x \in \mathbb{R}^{S} \mid x(S) \leqslant 0\right\}$ so that (5.1) - (5.5) are satisfied.

(4) Otherwise, $S=P \cup Q$ for some $P \in \mathcal{F}^{N}$ and some $Q \in \mathcal{F}^{M \backslash N}$. In this case define

$$
W(S)=\left\{(x, y)-y(Q) \chi^{P, S} \mid x \in V(P), y \in \mathbb{R}^{Q}\right\} .
$$

Then it is straightforward to verify that $W(S)$ is nonempty, closed, comprehensive, and non-leveled. If $z \in \mathbb{R}^{S}$ and $y \in W(S) \cap\left(z+\mathbb{R}_{+}^{S}\right)$, then $y_{Q} \geqslant z_{Q}$, hence $y(Q) \geqslant z(Q)$. As $y_{P}+y(Q) \chi^{P, P}=x \in$ $V(P), x \geqslant z_{P}+z(Q) \chi^{P, P}$ so that (5.4) follows. We'll use the obvious fact that

$$
\left\{x \in \mathbb{R}^{P} \mid\left(x, 0_{Q}\right) \in W(S)\right\}=V(P) .
$$


In order to complete the proof, let $y \in C(M, W, h)$. By construction $M / h=\{R \cup\{j(R)\} \mid R \in \mathcal{T}\}$. Let $R \in \mathcal{T}$ and $j=j(R)$. By case (4) there exists $x \in V(R)$ such that $y_{i}=x_{i}-y_{j}$ for all $i \in R$. By (3), $y_{j} \geqslant 0$. As $x \in W(R)$ by (2), $y_{j}=0$. By $(5.10), x_{R}=\bar{x}_{R}$ hence, $y=\left(\bar{x}, 0_{M \backslash N}\right)$. We conclude that $C(M, W, h)=\{y\}$ and $W_{h}^{N, y}=V$. As $h^{N}=g$, the proof is complete.

Now we are able to prove the following theorem.

Theorem 5.4 The core on $\Delta_{U}^{C}$ is characterized by

(1) NE, IR, WRGP, and CRGP, provided that $|U| \geqslant 3$, and by

(2) NE, IR, and RGP, provided that $|U|=\infty$.

Proof: By definition of $\Delta_{U}^{C}$ the core satisfies NE, and by Remark 5.1 it satisfies the remaining properties. In order to show uniqueness, let $\sigma$ be a solution that satisfies the desired properties and let $(N, V, g) \in \Delta_{U}^{C}$. By Lemma 5.2, $\sigma(N, V, g) \subseteq C(N, V, g)$ so that only the other inclusion remains to be verified. If $|N| \leqslant 2$, then $|\{R \in N / g|2 \leqslant| R \mid\}| \in\{0,1\}$. By Lemma 5.2, Lemma 5.3, WRGP, and NE the proof is finished in this case because $|U| \geqslant 3$. Now, let $|N| \geqslant 3$ and $x \in C(N, V, g)$. By WRGP of $C$, $x_{S} \in C\left(S, V_{g}^{S, x}\right)=\sigma\left(S, V_{g}^{S, x}\right)$ for all $S \subseteq N$ with $|S|=2$ and $\left|S / g^{S}\right|=1$. If $\sigma$ satisfies CRGP, then $x \in \sigma(N, V, g)$. If $|U|=\infty$, then the proof is complete by NE, RGP, and Lemmas 5.2 and 5.3.

\subsection{Theorems 3.1 and 3.5 for the NTU case}

This subsection is devoted to generalize Theorem 7.4 and 7.5 of Hwang and Sudhölter (2001) in order to suitably modify Theorems 3.1 and 3.5 for NTU games with communication structures. We first generalize some possible properties of a solution. Let $(N, V, g) \in \Delta_{U}, \widehat{S} \in \mathcal{F}^{N}, X \subseteq \mathbb{R}^{\widehat{S}}$, and $a \in \mathbb{R}^{N}$. Then we denote $a * X=\left\{\left(a_{i} x_{i}\right)_{i \in \widehat{S}} \in \mathbb{R}^{\widehat{S}} \mid x \in X\right\}$ and call $(N, V, g)$ a-inessential if $a_{S} \in \partial V(S)$ for all $S \in \mathcal{F}^{N}$. A solution $\sigma$ on $\Delta$ satisfies

- COV if for all $(N, V, g),(N, W, g) \in \Delta_{U}$ such that $W=\alpha * V+\beta$ for some $\alpha, \beta \in \mathbb{R}^{N}$ with $\alpha \gg 0$, $\sigma(N, W, g)=\alpha * \sigma(N, V, g)+\beta$

- ZIG if for every 0-inessential 2-person game $(N, V, g) \in \Delta_{U}, \sigma(N, V, g) \neq \emptyset$;

- RCP if the following condition is satisfied for every $(N, V, g) \in \Gamma, x \in \sigma(N, V, g)$, and $S \in \mathcal{F}^{N}$ : If $\left(S, V_{g}^{S, x}, g^{S}\right) \in \Gamma$ and $y \in \sigma\left(S, v_{g}^{S, x}, g^{S}\right)$ satisfies, for all $R \in S / g^{S}$,

$$
\begin{array}{r}
\bigcup_{Q \subseteq N \backslash R, R \cup Q \in \mathcal{F}_{g}^{N}}\left\{z \in \mathbb{R}^{R} \mid z \gg 0,\left(y_{R}+z, x_{Q}\right) \in V(R \cup Q)\right\} \\
\subseteq \bigcup_{Q \subseteq N \backslash R, R \cup Q \in \mathcal{F}_{g}^{N}}\left\{z \in \mathbb{R}^{R} \mid z \gg 0,\left(x_{R}+z, x_{Q}\right) \in V(R \cup Q)\right\},
\end{array}
$$

then $\left(y, x_{N \backslash S}\right) \in \sigma(N, V, g)$;

- REAS if for every $(N, V, g) \in \Delta, x \in \sigma(N, V, g)$, and all $i \in N$,

$x_{i} \geqslant d_{i}(N, V, g)=\min \left\{\max \left\{t \in \mathbb{R}^{\{i\}} \mid(t, y) \in V(S \cup\{i\}) \forall y \in V(S)\right\} \mid S, S \cup\{i\} \in \mathcal{F}_{g}^{N} \cup\{\emptyset\}, S \not \ngtr i\right\}$,

where $V(\emptyset)=\mathbb{R}^{\emptyset}$ and $\max \emptyset=-\infty$ (in this case $x$ is called reasonable). 
Remark 5.5 (1) It should be noted that, if $(N, V)$ corresponds to a TU game, then (5.12) is equivalent to the condition $y(R) \geqslant x(R)$ (see the definition of RCP in Sect. 3). Originally (Hwang and Sudhölter 2001) these conditions were not included in the definitions of RCP so that the current versions were called weak RCP (for interpretations see the aforementioned paper). For TU games, the core satisfies even the stronger property (where $y(R) \geqslant x(R)$ is not assumed) because $y(R)=$ $x(R)$ whenever $x_{S}$ and $y_{S}$ belong to the core of the reduced TU game with communication structure. In view of Lemma 5.3, for NTU games the core does not satisfy the stronger property because connected coalitions of the form $R \cup Q$ might be able to improve upon $\left(y, x_{N \backslash S}\right)$ which is ruled out for a core element $x$ if $y$ satisfies $(5.12)$ because $x_{S}$ is in the core of $\left(S, V_{g}^{S, x}, g^{S}\right)$. Hence, the core satisfies the current version of RCP.

(2) Let $(N, V, g) \in \Delta_{U}$. Then $X=\{x \in X(N, V, g) \mid x$ is reasonable $\} \neq \emptyset$. Indeed, denote $S=\{i \in$ $\left.N \mid d_{i}(N, v, g)=-\infty\right\}$ and choose any $y \in \mathbb{R}^{N}$ that satisfies $y_{j}=d_{j}(N, V, g)$ for all $j \in N \backslash S$ and $y_{P} \in V(P)$ for all $P \in \mathcal{F}^{S}$. Let $T \in \mathcal{F}_{g}^{N}$. By induction on $|T|$ we show that $y_{T} \in V(T)$. If $|T|=1$, then $y_{T} \in V(T)$ by construction. If $|T| \geqslant 2$, then there exists $i \in T$ such that $T \backslash\{i\} \in \mathcal{F}_{g}^{N}$. By the inductive hypothesis $y_{T \backslash\{i\}} \in V(T \backslash\{i\})$ so that $y_{T} \in V(T)$ by construction. By (5.3), there exists $x \in X(N, v, g)$ such that $x \geqslant y$ so that $x$ is reasonable. Note that the core satisfies REAS.

(3) Note that the set of reasonable Pareto optimal payoff vectors (i.e., the set $X$ defined in (2)) may be indeed unbounded. E.g., if $|N|=2, N \in g, \max V(\{i\})=0$ for $i \in N$, and $V(N)$ is contained in $\left\{x \in \mathbb{R}^{N} \mid x \ll 0\right\}$, then $d_{i}(N, V, g)=-\infty$.

For $(N, V, g) \in \Delta_{u}$ and $S \in \mathcal{F}^{N}$ the subgame with communication structure on $S$ is denoted by $(S, V, g)$. As in the TU context, the Myerson restricted game with communication structure of $(S, V, g)$ coincides with the subgame with communication structure of $(N, V / g)$ on $S$ so that it is denoted by $(S, V / g)$. We say that $(N, V, g)$ is (totally) cardinal balanced if $(N, V / g)$ is (totally) cardinal balanced in the sense of Billera and Bixby (1973). A cardinal balanced NTU game has a nonempty core, but there are NTU games with a nonempty core that do not even satisfy the weaker balance property of Scarf (1967). However, for 2-person NTU games these conditions are also necessary for the non-emptiness of the core. Let $\Delta_{U}^{t b}$ denote the set of totally cardinal balanced NTU games with communication structures.

Theorem 5.6 If $|U| \geqslant 5$ and $\Delta_{U}^{t b} \subseteq \Delta \subseteq \Delta_{U}$ such that any $(N, v, g) \in \Gamma$ with $|N|=2$ is cardinal balanced, then the core

(1) on $\Delta_{U}$ is characterized by ZIG, AN, COV, WRGP, RCP, CRGP, and REAS;

(2) on $\Delta$ is characterized by ZIG, COV, WRGP, RCP, CRGP, and REAS.

Proof: The core satisfies the desired properties so that it remains to show the uniqueness part. Let $\sigma$ be a solution on $\Delta_{U}$ or $\Delta$, respectively, that satisfies the respective desired properties and let $(N, V, g)$ be an element of $\Delta_{U}$ or $\Delta$, respectively. By Theorem 7.4 or Theorem 7.5 of Hwang and Sudhölter (2001), $\sigma(N, V, g)=C(N, V, g)$ if $(N, g)$ is complete. Hence, we may assume that $(N, g)$ is not complete.

If $|N|=2$, then $\sigma(N, V, g)$ is contained in the singleton core by REAS. Hence, it suffices to show that $\sigma(N, V, g) \neq \emptyset$. Choose $\ell \in U \backslash N$ and $k \in N$ and define $M=N \cup\{\ell\}$ and $(M, W, h)$ by $h=\{\{k, \ell\}\}$, $W(S)=V(S)$ and $W(S \cup\{\ell\})=\left\{\left(x+t \chi^{S, N},-t\right) \in \mathbb{R}^{S \cup\{\ell\}} \mid x \in V(S), t \in \mathbb{R}^{\{l\}}\right\}$ for $S \in \mathcal{F}^{N}$, 
and $W(\{\ell\})=-\mathbb{R}_{+}^{\{\ell\}}$. Then $(M, W / h)$ corresponds to a TU game and, with $x \in \mathbb{R}^{M}$ given by $x_{i}=$ $\max W(\{i\})$ for $i \in M, x \in C(M, W, h)$ so that $(M, W, h)$ is cardinal balanced. Moreover, for $S \subseteq M$ with $1 \leqslant|S|=2, x_{S} \in C(S, W, h)$. Hence, $(M, W, h)$ is totally balanced. By CRGP, $x \in \sigma(M, W, h)$ because the unique relevant reduced game is the reduced game on $\{k, \ell\}$. Hence, by WRGP, $x_{N} \in \sigma(N, V, g)$. If $|N| \geqslant 3$ and $x \in \sigma(N, V, g)$, then by WRGP, $x$ is Pareto optimal, hence $\sigma$ satisfies PO. Thus, the appropriate generalization of Remark 3.3 (1) to NTU games with communication structures finishes the proof.

For a single NTU game Keiding and Thorlund-Petersen (1987) and more recently Predtetchinski and Herings (2004) present necessary and sufficient conditions for non-emptiness of the core that, by (5.7), can also be applied to NTU games with communication structures. It should be remarked that Theorem 5.4 (1), though it does not provide a statement about the non-emptiness of the core of a single game, yields an implicit characterization of the set of NTU games with communication structures that have a nonempty core.

\section{The logical independence of the employed axioms}

By generalizing or modifying if necessary the relevant examples of the literature we show that each axiom employed in any of the foregoing theorems and in Corollary 4.5 is logically independent of the remaining axioms.

Keeping the names of the solutions assigned by Hwang and Sudhölter (2001, Sect. 8) we define, for any $(N, V, g) \in \Delta_{U}$,

$$
\begin{aligned}
& \sigma^{2}(N, V, g)= \begin{cases}C(N, V, g) & , \text { if }|N| \geqslant 2, \\
V(N) & , \text { if }|N|=1\end{cases} \\
& \sigma^{3}(N, V, g)= \begin{cases}C(N, V, g) & , \text { if }(N, V) \text { is inessential, } \\
\emptyset & \text {, otherwise; }\end{cases} \\
& \sigma^{4}(N, V, g)=\left\{y \in C(N, V, g)\left|y_{S} \in \sigma^{3}\left(S, V_{g}^{S, y}, g^{S}\right) \forall S \subseteq N,\right| S \mid=2\right\} ; \\
& \sigma^{5}(N, V, g)=\left\{y \in X(N, V, g) \mid y_{i} \geqslant \max V(\{i\}) \text { for all } i \in N\right\} \text {; } \\
& \sigma^{6}(N, V, g)= \begin{cases}\{0\} & , \text { if } 0 \in C(N, V, g), \\
\emptyset & , \text { otherwise. }\end{cases}
\end{aligned}
$$

The "empty solution" satisfies all properties in all results except NE in Theorems 5.4 and 4.2 and in Corollary 4.5 and except ZIG in Theorems 5.6, 3.5, and 3.1. Moreover, $\sigma^{2}$ satisfies all axioms in the mentioned results except BOUND or REAS, respectively, $\sigma^{3}$ exclusively violates CRGP in all results except in Theorem 5.4 (1), $\sigma^{4}$ exclusively violates RCP wherever RCP is employed, $\sigma^{5}$ exclusively violates RGP and WRGP, respectively, and $\sigma^{6}$ exclusively violates COV. The following solution $\sigma^{7}$ exclusively violates AN in Theorems 5.6 (1) and 3.1: Choose $\widetilde{N} \subseteq U$ with $|\widetilde{N}|=2$, let $(\widetilde{N}, U)$ be the NTU game corresponding to the negative of the unanimity TU game $(\widetilde{N}, u)$ given by $u(\widetilde{N})=-1$ and $u(S)=0$ for all $S \varsubsetneqq \widetilde{N}$, and let $z \in \mathbb{R}^{\widetilde{N}}$ be given by $z_{i}=-\frac{1}{2}$ for $i \in \widetilde{N}$ (i.e., $z$ is the standard solution of $(N, U)$ ). 
Now, define

$\sigma^{7}(N, V, g)= \begin{cases}\{a * z+\beta\} & , \text { if } N=\tilde{N} \in g \text { and there exist } \alpha, \beta \in \mathbb{R}^{N} \text { with } \alpha \gg 0 \text { and } V=\alpha * U+\beta, \\ C(N, V, g) & , \text { otherwise. }\end{cases}$

In order to show that SUPA is logically independent of the remaining axioms in all results that employ SUPA, we generalize Peleg's (1986) example: For every TU game with communication structure $(N, v, g) \in \Gamma_{U}$ the prekernel (Maschler, Peleg, and Shapley 1972, Davis and Maschler 1965) of $(N, v, g)$, denoted $\mathcal{P} \mathcal{K}(N, v, g)$, is defined by

$$
\mathcal{P} \mathcal{K}(N, v, g)=\left\{x \in X(N, v, N / g) \mid s_{k \ell}(x, v, g)=s_{\ell k}(x, v, g) \text { for all } k, \ell \in R \in N / g, k \neq \ell\right\},
$$

where $s_{i j}(y, v, g)=\max \left\{v(S)-y(S) \mid S \in \mathcal{F}_{g}^{N}, k \in S \not \supset \ell\right\}$ for $y \in \mathbb{R}^{N}$. As for classical TU games, $\mathcal{P K}(N, v, g)$ contains the prenucleolus (Schmeidler 1969, Sobolev 1975) point $\nu(N, v, g)$, and, if $(N, v, g)$ is balanced, then ${ }^{2} \nu(N, v, g) \in C(N, v, g)$. Hence, with

$$
\sigma^{0}(N, v, g)=\mathcal{P} \mathcal{K}(N, v, g) \cap C(N, v, g)
$$

the solution $\sigma^{0}$ satisfies NE on any subset of $\Gamma_{U}^{b}$. As $\mathcal{P} \mathcal{K}$ satisfies, among other properties, RGP and WRGP when possible and CRGP on every set of TU games with communication structures (Khmelnitskaya and Sudhölter 2013, Sect. 6), $\sigma^{0}$ exclusively violates SUPA wherever SUPA is employed.

Peleg (1985, Example 5.5) presents a solution $\sigma$ that satisfies NE, IR, WRGP, and SUPA on the set of totally balanced TU games $(N, v) \in \Gamma_{U}$, but does not coincide with the core, and coincides with $C(N, v)$ for all $(N, v) \in \Gamma_{U}^{b}$ with $|N| \leqslant 2$. If we generalize this solution by defining $\sigma^{\prime}(N, v, g)=\sigma(N, v)$ if $(N, g)$ is complete and $(N, v)$ is totally balanced, and $\sigma^{\prime}(N, v, g)=C(N, v, g)$ otherwise, then $\sigma^{\prime}$ exclusively violates CRGP in Corollary 4.5 and Remark 4.1.

It should be noted that the assumptions $|U| \geqslant 5,|U| \geqslant 4,|U| \geqslant 3$, and $|U|=\infty$, respectively, are sharp in the corresponding characterizations of the core in the sense that the results are no longer valid if $|U|=4,|U|=3,|U|=2$, and $|U|$ is finite and sufficiently large, respectively. E.g., $\sigma^{4}(N, V, g)$ is empty or a singleton for any 3-person game $(N, V, g) \in \Delta_{U}$ so that $\sigma^{4}$ also satisfies RCP if $|U|=4$.

\section{Concluding remarks}

We first remark that Peleg's (1989) Theorem 7.5 may be generalized to games with communication structures as the following corollary shows. Let $(N, v, g) \in \Gamma_{U}$ and $k, \ell \in N, k \neq \ell$. We say that $k$ and $\ell$ are substitutes w.r.t. $(N, v, g)$ if, for all $S \subseteq N \backslash\{k, \ell\}$, (a) $v(S \cup\{k\})=v(S \cup\{\ell\})$ and (b) $S \cup\{k\} \in \mathcal{F}_{g}^{N}$ if and only if $S \cup\{\ell\} \in \mathcal{F}_{g}^{N}$. Note that (b) is equivalent to the requirement that, for any $j \in N \backslash\{k, \ell\}$, $\{j, k\} \in g$ if and only if $\{j, \ell\} \in g$. A solution $\sigma$ on some $\Gamma \subseteq \Gamma_{U}$ satisfies the restricted equal treatment property $(\mathrm{RETP})$ if, for any $(N, v, g) \in \Gamma$ and any substitutes $k, \ell \in N$ w.r.t. $(N, v, g), x_{k}=x_{\ell}$ for all $x \in \sigma(N, v, g)$.

Corollary 7.1 The intersection of the prekernel and the core, $\sigma^{0}$, on $\Gamma_{U}^{b}$ is characterized by $\mathrm{NE}, \mathrm{COV}$, RETP, IR, WRGP, and CRGP.

\footnotetext{
${ }^{2}$ Note that $\nu(N, v, g)$ may not coincide with $\nu(N, v / g)$ in general already for $|N|=3$ as shown by an example (Khmelnitskaya and Sudhölter 2013, p. 297), but using Kohlberg's (1971) characterization of the (pre)nucleolus by balanced collections of coalitions it may be deduced that $\nu(N, v, g)=\nu(N, v / g)$ for all balanced $(N, v, g)$.
} 
Secondly we remark that, wherever it occurs, CRGP may be replaced by the weaker version that differs from CRGP only inasmuch as " $|N|-|N / g| \geqslant 1$ " is replaced by " $|N| \geqslant 2$ " and "and $k, \ell \in R$ for some $R \in N / g$ " is deleted in the corresponding definition, i.e., in order to enforce a preimputation $x$ to be a member of the solution, it is required that its restriction $x_{S}$ to any two-person coalition $S$ must be a member of the solution of the corresponding reduced game. In view of the fact that the members of two-person coalitions that are not connected in the reduced graph cannot communicate with each other, the original stronger property seems to be more reasonable. However, when using the aforementioned weaker property, only the proof of Lemma 3.4 becomes slightly more sophisticated.

Finally we remark that all results of the foregoing sections may be expanded to games with conference structures à la Myerson (1980). A conference structure is a pair $(N, \mathcal{Q})$ where $N \in \mathcal{F}$ and $\mathcal{Q} \subseteq 2^{N}$ satisfies $|Q| \geq 2$ for all $Q \in \mathcal{Q}$. An NTU game with conference structure is a triple $(N, V, \mathcal{Q})$ such that $(N, V) \in \Delta_{U}$ and $(N, \mathcal{Q})$ is a conference structure. As Khmelnitskaya and Sudhölter (2013) we identify a graph $(N, g)$ with the conference structure $(N, \mathcal{Q}(g))$ of all coalitions that contain at least two elements and are connected by $g$.

Let $(N, \mathcal{Q})$ be a conference structure and $S \subseteq N$. Recall that $i$ and $j$ are connected in $S$ by $\mathcal{Q}$ if $i, j \in S$ and there exists a chain $\left(Q_{1}, \ldots, Q_{\ell}\right)$ in $\mathcal{Q}$, i.e., $Q_{1}, \ldots, Q_{\ell} \in \mathcal{Q}$ and $Q_{t} \cap Q_{t+1} \neq \emptyset$ for all $t=1, \ldots, \ell-1$, such that $i \in Q_{1}, j \in Q_{\ell}$, and $Q_{t} \subseteq S$ for $t=1, \ldots, \ell$. Moreover, $S / \mathcal{Q}$ denotes the set of components of $S$ w.r.t. $\mathcal{Q}$, and a coalition $S$ that has only one component is called connected. Furthermore, $\mathcal{F}_{\mathcal{Q}}^{N}$ denotes the set of all coalitions in $N$ that are connected by $\mathcal{Q}$. For $S \in \mathcal{F}^{N}$ the reduced conference structure $\left(S, \mathcal{Q}^{S}\right)$ w.r.t. $S$ is defined by

$$
\mathcal{Q}^{S}=\left\{S \cap \bigcup_{t=1}^{\ell} Q_{t} \mid \ell \in \mathbb{N}, Q_{t}, Q_{\ell} \in \mathcal{Q}, \emptyset \neq Q_{t} \cap Q_{t+1} \subseteq N \backslash S \forall t \in \mathbb{N} \text { with } t<\ell,\left|S \cap \bigcup_{t=1}^{\ell} Q_{t}\right| \geqslant 2\right\},
$$

that is, $Q^{\prime} \subseteq S$ is a conference in $\mathcal{Q}^{S}$ if $\left|Q^{\prime}\right| \geqslant 2$ and $Q^{\prime}$ is the intersection with $S$ of the union of all members of a chain of conferences in $\mathcal{Q}$ such that each $i \in Q^{\prime}$ is an element of a unique member of this chain. Note that the definition given in (7.1) differs from the definitions of a reduced conference structure provided by Albizuri and Zarzuelo (2009, equation (7)) or by Khmelnitskaya and Sudhölter (2013, equation (7.2)), but the arising sets of connected coalitions in the reduced conference structures coincide for the aforementioned three distinct definitions. We think, however, that (7.1) reflects better that each player of $Q^{\prime}$ can only evoke a unique element of the chain of conferences, whereas outside of $S$ each conference is able to join. E.g., if $N=\{1, \ldots, 4\}, \mathcal{Q}=\{\{1,2\},\{2,3,4\}\}$, and $S=\{1,2,3\}$, then $\mathcal{Q}^{S}=\{\{1,2\},\{2,3\}\}$, whereas according to the two mentioned definitions $Q^{\prime}=\{1,2,3\}$ would be a further element of the reduced conference structure. This "conference" can only be created if the two distinct conferences $\{1,2\}$ and $\{2,3,4\}$ of $\mathcal{Q}$ can be evoked by one player of $Q^{\prime}$ (namely player 2) which seems unreasonable.

Note that $\mathcal{Q}^{N}=\mathcal{Q}$ and $\mathcal{F}_{\mathcal{Q}^{S}}^{S}=\left\{T \cap S \mid T \in \mathcal{F}_{\mathcal{Q}}^{N}\right\} \backslash\{\emptyset\}$ for any conference structure $(N, \mathcal{Q})$ and $S \in \mathcal{F}^{N}$. Note also that $(\mathcal{Q}(g))^{S}=\mathcal{Q}\left(g^{S}\right)$ and $\mathcal{F}_{g}^{N}=\mathcal{F}_{\mathcal{Q}(g)}^{N}$ for each communication structure $(N, g)$. Now, all the definitions of the preceding sections may be generalized when communication structures are replaced by conference structures. Basically, the character $g$ denoting a graph has to be replaced by the character $\mathcal{Q}$ denoting a conference structure. All previous results hold for games with conference structures and most of the corresponding proofs can be literally copied. The other proofs have to be slightly modified in a straightforward way. 


\section{References}

Albizuri, M. J., And J. M. Zarzuelo (2009): "Conference structures and consistency", Discrete Mathematics, 309, $4969-4976$.

Billera, L. J., And R. E. Bixby (1973): "A characterization of polyhedral market games", International Journal of Game Theory, 2, $253-261$.

Bondareva, O. N. (1963): "Some applications of linear programming methods to the theory of cooperative games", Problemi Kibernitiki, 10, 119-139.

Davis, M., And M. Maschler (1965): "The kernel of a cooperative game", Naval Research Logistics Quarterly, 12, 223-259.

Faigle, U. (1989): "Cores of games with restricted cooperation", Zeitschrift für Operations ResearchMathematical Methods of Operations Research, 33, 405 - 422.

Herings, P., G. van der laAn, A. Talman, and Z. Yang (2010): "The average tree solution for cooperative games with communication structure", Games and Economic Behavior, 68, 626 - 633.

HWANG, Y.-A., AND P. SUdhöLter (2001): "Axiomatizations of the core on the universal domain and other natural domains", International Journal of Game Theory, 29, 597-623.

Keiding, H., and L. Thorlund-Petersen (1987): "The core of a cooperative game without side payments", Journal of Optimization Theory and Applications, 54, $273-288$.

Khmelnitskaya, A., And P. Sudhölter (2013): "The prenucleolus and the prekernel for games with communication structures", Mathematical Methods of Operations Research, 78, 285 - 299.

Maschler, M., B. Peleg, and L. S. Shapley (1972): "The kernel and bargaining set for convex games", International Journal of Game Theory, 1, 73 - 93.

Myerson, R. B. (1977): "Graphs and cooperation in games", Mathematics of Operations Research, 2, $225-229$.

9, 169 - 1820):

PeleG, B. (1985): "An axiomatization of the core of cooperative games without side payments", Journal of Mathematical Economics, 14, 203 - 214.

(1986): "On the reduced game property and its converse", International Journal of Game Theory, 15, $187-200$.

(1989): "An axiomatization of the core of market games", Mathematics of Operations Research, $14,448-456$.

Predtetchinski, A., And P. J.-J. Herings (2004): "A necessary and sufficient condition for nonemptiness of the core of a non-transferable utility game", Journal of Economic Theory, 116, 84-92.

SCARF, H. E. (1967): "The core of an n-person game", Econometrica, 35, 50 - 69.

Schmeidler, D. (1969): "The nucleolus of a characteristic function game", SIAM Journal on Applied Mathematics, 17, 1163 - 1170.

Serrano, R., and O. VoliJ (1998): "Axiomatizations of neoclassical concepts for economies", Journal of Mathematical Economics, 30, 87 - 108.

ShaPley, L. S. (1967): "On balanced sets and cores", Naval Research Logistics Quarterly, 14, 453 460. 
Sobolev, A. I. (1975): "The characterization of optimality principles in cooperative games by functional equations", in Mathematical Methods in the Social Sciences, ed. by N. N. Vorobiev, Vol. 6, pp. 95 151, Vilnius. Academy of Sciences of the Lithuanian SSR, in Russian.

Sudhölter, P., ANd B. Peleg (2002): "A note on an axiomatization of the core of market games", Mathematics of Operations Research, 27, $441-444$.

TADEnuma, K. (1992): "Reduced games, consistency, and the core", International Journal of Game Theory, 20, $325-334$. 